Check for updates

Cite this: RSC Adv., 2017, 7, 37076

Received 19th May 2017

Accepted 20th July 2017

DOI: $10.1039 / \mathrm{c} 7 \mathrm{ra0} 5664 \mathrm{~h}$

rsc.li/rsc-advances

\section{Optical-signal-enhancing metasurface platforms for fluorescent molecules at water-transparent near-infrared wavelengths $\uparrow$}

\author{
Hiroyuki Kurosawa and Masanobu Iwanaga (iD *
}

\begin{abstract}
We report efficient sensing platforms to obtain artificially enhanced optical signals from near-infrared fluorescent molecules with emitting wavelengths in $1.1 \mu \mathrm{m}$ range, which corresponds to the so-called "living-body window". Prominent enhancement was experimentally achieved employing high-emittance metasurfaces of plasmo-photonic resonances. In addition, we show that enhanced fluorescence and Raman scattering are selectable using the metasurfaces with and without a self-assembled monolayer, which determines the contact of the molecules with outmost surface of noble metals. We also experimentally studied the effect of $\mathrm{Au}$ and $\mathrm{Ag}$ on the enhancement of optical signals, and numerically examined the conditions for attaining optimal enhancement. This paper describes a systematic study on artificially enhanced HOMO-LUMO transitions in the fluorescent molecules, explicitly suggesting an effective way to attain prominent enhancement for low-quantum-yield fluorescent molecules.
\end{abstract}

\section{Introduction}

Most of the existing molecular sensing techniques have been motivated to apply them for biosensing. For efficient bioimaging of living tissues, one of the most important wavelength ranges is $1.1 \mu \mathrm{m}$ band, which is often called "living-body window", since the light absorption by water and hemoglobin is minimal in the window. However, molecular sensing techniques based on plasmonic enhancement typically use fluorescent molecules that emit light with wavelengths shorter than $850 \mathrm{~nm} .^{1,2}$ Indeed, metallic nanoparticle-based ${ }^{1-8}$ or nanogap-based ${ }^{\mathbf{1 , 2 , 9 - 1 5}}$ plasmonic enhancement of fluorescence intensity is mostly limited to wavelengths in the $600-800 \mathrm{~nm}$ range. This is partially because plasmonic structures, which are highly efficient with respect to enhancement of optical signals and simultaneously flexible with respect to their working wavelengths, have been hardly known.

In contrast, there are virtually no reports to show efficient optical-signal enhancement of fluorescent molecules working in the $1.1 \mu \mathrm{m}$ band. ${ }^{16}$ Generally, fluorescent molecules in the 1.1 $\mu \mathrm{m}$ band exhibit lower fluorescence yield than those in the visible wavelengths; therefore, it is difficult to attain prominent fluorescence enhancement using plasmonic structures composed of metallic nanostructures; to the best of our

National Institute for Materials Science (NIMS), 1-1 Namiki, Tsukuba 305-0044, Japan. E-mail: iwanaga.masanobu@nims.go.jp

$\dagger$ Electronic supplementary information (ESI) available: SEM images to confirm the stacked complementary structure; measured reflectance spectra of $\mathrm{Au}$ and Ag SC PlasPh metasurfaces; enhanced optical signal with wavenumber representation; measured data on an IR26-dispersed $\mathrm{SiO}_{2}$ substrate; numerically calculated resonant electric fields of $\mathrm{Ag}$ PlasPh metasurface; measured and cited data on complex permittivity of Au and Ag. See DOI: 10.1039/c7ra05664h knowledge, fluorescence enhancement has not been reported for the $1.1 \mu \mathrm{m}$ band. As for other luminescent materials, there are a few reports addressing metal-induced photoluminescence enhancement of $\mathrm{Ag}_{2} \mathrm{~S}$ quantum dots ${ }^{17}$ and carbon nanotube ${ }^{18}$ at the $1.1 \mu \mathrm{m}$ band; the maximum photoluminescence enhancement was not so large, and at most 100 -fold ${ }^{17}$ and 10 -fold ${ }^{18}$ for relevant references. The luminescent materials working at the $1.1 \mu \mathrm{m}$ band are potential to optical sensing and/or imaging in aqueous circumstances including biotissues.

Thus, there is enough room to explore other platforms which show highly enhancing optical signals of fluorescent molecules or other luminescent materials in the $1.1 \mu \mathrm{m}$ band. Considering the present status, we designed optical experiment to show prominent enhancement of optical signals such as fluorescence and Raman scattering in the $1.1 \mu \mathrm{m}$ band. The present platform is particularly suitable to in situ sensing of molecules working in aqueous circumstances like proteins.

Stacked complementary (SC) plasmo-photonic (PlasPh) metasurfaces $^{\mathbf{1 9 , 2 0}}$ are considered as good optical-signal enhancement platforms, which are directly applicable to molecular sensing. This metasurface is fabricated using a silicon-on-insulator (SOI) substrate, forms a SC noble-metal structure, and exhibits a series of hybrid plasmon-photonicguided-mode resonances. The PlasPh metasurfaces have been reported to have tunable resonances in a wide wavelength range from the visible to the near-infrared telecom ranges. ${ }^{20,21}$ The PlasPh metasurfaces have been found to be highly suitable for enhanced spectroscopy of fluorescent molecules around the visible range. ${ }^{20-23}$ For other metallic nanostructures, it is usually quite demanding to ensure tunability of the operating wavelength and, at the same time, capability for high-efficient 
enhancement. In this respect, photonic-crystal-based platforms ${ }^{24}$ are different, because they allow to scale the operating wavelengths, though photonic crystals tend to show weaker enhancement than plasmonic platforms.

Here, we report experimental observations of optical-signal enhancement using IR26 molecules (Exciton Inc., Ohio, USA), which work mainly in the living-body window. In addition, we compare the SC PlasPh metasurfaces incorporating $\mathrm{Au}$ and $\mathrm{Ag}$, and address the difference. We also performed numerical analysis of the enhancement mechanism, and discuss the experimental results based on the numerical findings.

\section{Results and discussion}

Fig. 1 shows basic properties of the IR26 fluorescent molecules. Extinction and fluorescence spectra were first reported in $1980 \mathrm{~s},{ }^{25}$ showing the fluorescence and lasing in the living-body window. Fig. 1a shows a photograph of an IR26 methanol solution of $70 \mu \mathrm{M}$; the solution is dark. IR26 molecules are partially soluble in organic solvents such as methanol, ethanol, and acetyl glycol; as a result, a fraction of the molecules remains to be in the form of powder in the solution. Fig. 1b shows the structure of an IR26 molecule, which is slightly modified from the original report ${ }^{25}$ in terms of the attendant halide.

Fig. 1c shows the measured extinction spectrum of an IR26 solution of $50 \mu \mathrm{M}$ (black curve), measured using a quartz cuvette with the light path of $1 \mathrm{~cm}$, and the fluorescence spectrum of the IR26 molecules dispersed on a Si wafer (red curve), measured after drying the methanol solution in air. Owing to the low quantum yield, the fluorescence intensity is quite weak and the signal-tonoise ratio is small. The fluorescence peaks in $1.1 \mu \mathrm{m}$ band and decreases as the emission wavelength increases. The red arrow indicates the excitation laser line at $1064.1 \mathrm{~nm}$, which is resonant to the HOMO-LUMO excitation of IR26 molecules. The fluorescence was measured using a laser-line cut filter; therefore, a sharp edge appears at $1100 \mathrm{~nm}$ in the fluorescence spectrum. The detailed measurement conditions are described in Section 4.1 and 4.2.

\subsection{Detection of enhanced optical signals}

2.1.1 Au SC PlasPh metasurfaces without SAM. Fig. 2a shows a three-dimensional (3D) illustration of a Au SC PlasPh metasurface and the coordinate axes. The SC structures were fabricated using silicon-on-insulator (SOI) substrates; hexagonal arrays of air holes were introduced on the SOI substrates using nanoimprint lithography (NIL) in a large area of $\mathrm{cm}^{2}$. The periodicity was fixed at $\mathbf{4 1 0 . 5} \mathrm{nm}$ in this study. The top layer was a perforated $\mathrm{Au}$ film, the middle was a Si photonic crystal slab, and the bottom comprised a $\mathrm{Si}$ photonic crystal slab and embedded Au disks. The thickness of Au was set to $35 \mathrm{~nm}$. The top and bottom layers were spatially separated by $165 \mathrm{~nm}$ due to the middle layer. The fabrication procedure using the NIL is described in Section 4.1. The SC structures were confirmed conducting section-view observation; the scanning-electronmicroscope (SEM) images are shown in Fig. S1 (ESI $\dagger$ ). The SC structure was isolated by buried oxide (BOX) from the base $\mathrm{Si}$ wafer of $625 \mu \mathrm{m}$ thickness, forming a waveguide structure. Incidence was set to travel from the $+z$ position and illuminate on the SC metasurface. The optical signals emitted from the IR26 molecules were collected using an objective lens (not drawn) that was located at the $+z$ position. Fig. 2 a also shows the molecular configuration on the metasurface. The dispersed IR26 molecules were directly placed on the outmost Au surface and possibly on the sidewall of air holes.

Fig. 2b illustrates the middle and bottom layers of the SC metasurface in Fig. 2a; dashed arrows from Fig. 2a indicate the correspondence. The two layers are displayed in a decomposed manner for clarity; the middle layer is a perforated Si crystal slab and the bottom is a Au-disk embedded Si slab.

Fig. 2c shows a top-view SEM image of a Au SC PlasPh metasurface with air-hole diameter $(D)$ of $212 \mathrm{~nm}$, which is indicated by a symbol $D$ 212; similar symbols are used in the following figures. The image was taken in a top-view configuration, observing the top layer from the air side.

Fig. 2d shows a typical measured optical signal (blue solid curve) emitted from the IR26 molecules dispersed on the Au SC PlasPh metasurface. The pumping wavelength was $1064.1 \mathrm{~nm}$, which is indicated by a red arrow. The signal was measured in the setup equivalent to that of the fluorescence measurement in Fig. 1c; consequently, a sharp edge at $1100 \mathrm{~nm}$ was observed, coming from the laser-line cut filter. The spectral shape of the measured optical signal basically originate from the fluorescence spectrum of IR26 molecules in Fig. 1c, whereas two prominent peaks appear around 1120 and $1170 \mathrm{~nm}$ in Fig. 2d. (a)

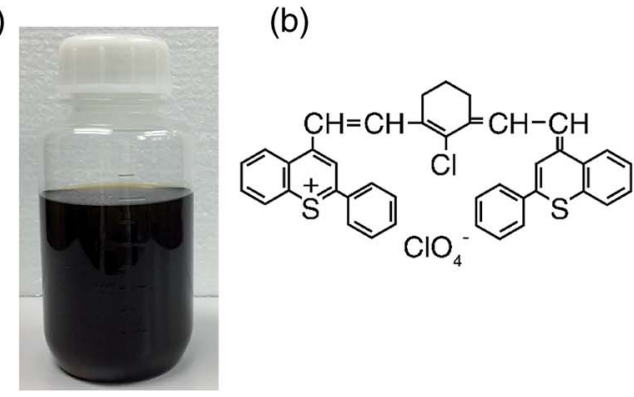

(c)

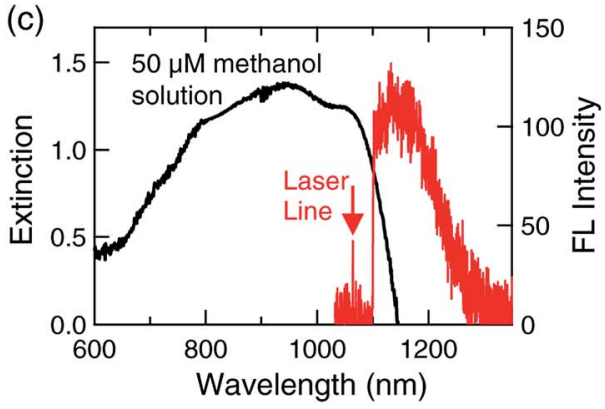

Fig. 1 (a) Appearance of the methanol solution of IR26 molecules $(70 \mu \mathrm{M})$. (b) The structure of an IR26 molecule. (c) Extinction spectrum (black) of the $50 \mu \mathrm{M}$ IR26 methanol solution. Fluorescence (FL) spectrum (red) of the IR26 molecules dispersed and dried on a Si wafer. The red arrow indicates the excitation laser line at $1064.1 \mathrm{~nm}$. 
(a)

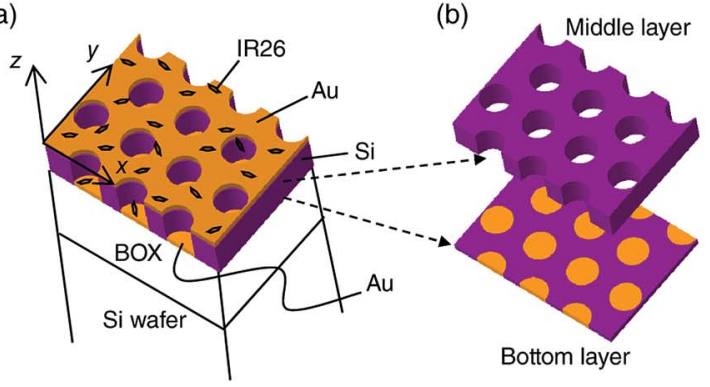

(c) Au SC PlasPh
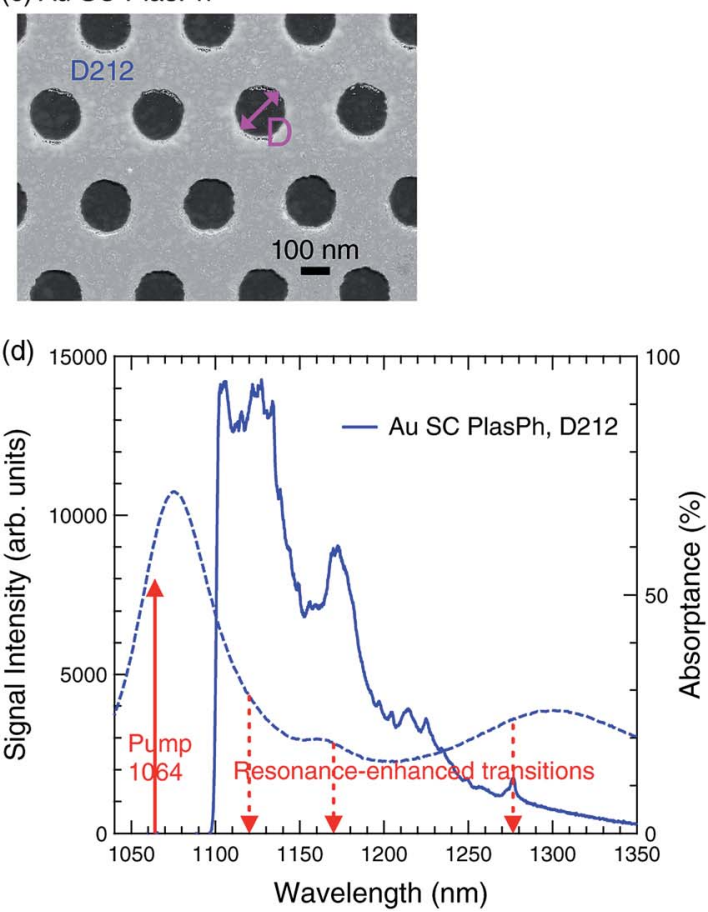

Fig. 2 (a) 3D-view schematic of a Au SC PlasPh metasurface, on which IR26 molecules are dispersed. The coordinate $x y z$ axes are set as shown. (b) The middle and bottom layers of the SC metasurface in (a) are illustrated in a decomposed way. (c) Top-view SEM image of a Au PlasPh metasurface of air-hole diameter D $212 \mathrm{~nm}$ (D 212). The scale bar indicates $100 \mathrm{~nm}$. (d) The measured enhanced optical signals (solid curve) and light absorptance spectrum (dashed curve), which is equivalent to the metasurface's emittance spectrum.

These peaks are associated with peaks of light absorptance (blue dashed curve). The absorptance was evaluated with equation such as

$$
A=100-R
$$

in \% where $A$ and $R$ denote the absorptance and reflectance, respectively. Note that the transmittance and diffraction are negligible in the wavelength range of present interest, not being accounted for in eqn (1). The measured reflectance spectra, shown in Fig. S2 (ESI $\dagger$ ), were used for evaluating the absorptance in eqn (1).

We point out that absorptance is equivalent to emittance from the metasurfaces, due to reciprocity; ${ }^{26}$ therefore, the prominent peaks are ascribed to emittance enhancement. Red dashed arrows indicate resonantly enhanced transitions. In addition to fluorescence, sharp 11 peaks also appear at 11101156, 1195-1227 and $1277 \mathrm{~nm}$ as shown in Fig. 2c; these peaks are attributed to the Raman scattering by the IR26 molecules from comparison with a previous report on the IR26 molecules. ${ }^{16}$ Wavenumber representation is generally used for Raman signals, being shown in Fig. S3 (ESI $\dagger$ ). We note that IR26 molecules have been rarely studied in plasmonic systems and the literature is very limited to our knowledge. As for the fluorescence enhancement, this paper is the first report.

2.1.2 Au SC PlasPh metasurfaces with SAM. Fig. 3a schematically shows experimental configuration in which an Au SC $\mathrm{PlasPh}$ metasurface is covered with a self-assembled monolayer (SAM). We used 15-carboxy-1-pentadecanethiol (15-CPDT, Dojindo, Kumamoto, Japan) as the SAM; the molecular structure is drawn in Fig. 3b. The SAM serves as spacer between the dispersed IR26 molecules and the outmost Au surface. Accounting for the length of the $\mathrm{C}-\mathrm{H}$ chain and the tilting angle on the Au surface, ${ }^{27}$ the thickness of the SAM is estimated to be $0.9 \mathrm{~nm}$.

Fig. 3c shows measured optical signals (solid curves) from the IR26 molecules dispersed on the Au SC PlasPh metasurfaces with the SAM. Three spectra are shown with blue, red and black, which were measured for the PlasPh metasurfaces with air-hole diameter $D$ 212, 220, and $280 \mathrm{~nm}$, respectively. Light absorptance spectra are also shown with dashed curves and the scale is shown on the right axis; the colors are similar to those of the optical signals. The absorptance spectra were evaluated using eqn (1), similarly to Fig. 2c. The measured reflectance spectra are shown in Fig. S2 (ESI $\dagger$ ). The red arrow indicates the

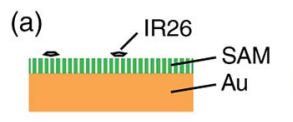

(b) 15-CPDT

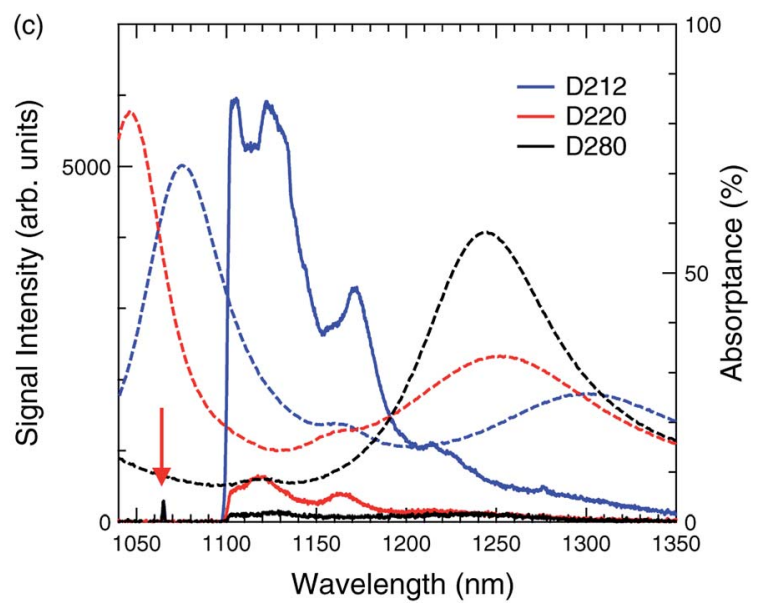

Fig. 3 (a) Schematic of molecules on the Au PlasPh metasurface with SAM. (b) The structure of a 15-CPDT molecule, forming the SAM. The $\mathrm{C}-\mathrm{H}$ chain stands on the Au surface with $\mathrm{Au}-\mathrm{S}$ covalent bond. (c) The measured optical signals (solid curves) and absorptance spectra (dashed curves). The blue, red, and black lines denote the PlasPh metasurfaces with air-hole diameter $D$ 212, 220 and $280 \mathrm{~nm}$, respectively. 
excitation wavelength of $1064.1 \mathrm{~nm}$, which is close to the resonance of the PlasPh metasurface with $D 212 \mathrm{~nm}$ and is off resonance to the PlasPh metasurface with $D 280 \mathrm{~nm}$.

In Fig. 3c, the strongest optical-signal enhancement was observed for the PlasPh metasurface with $D 212 \mathrm{~nm}$. This probably occurred because the excitation and emission wavelengths were matched for the resonances in the PlasPh metasurface with $D 212 \mathrm{~nm}$. Comparing the three cases of $D$ 212, 220 and $280 \mathrm{~nm}$, we find that it is primarily crucial whether the excitation wavelength is resonant or not for obtaining highly enhanced optical signals. Indeed, the PlasPh metasurface with $D 280 \mathrm{~nm}$ exhibited far weaker optical signals than the PlasPh metasurface with $D 212 \mathrm{~nm}$. This contrast strongly suggests that the excitation efficiency on the PlasPh metasurfaces directly contributes to the observed signal intensities.

As for the PlasPh metasurface with $D 220 \mathrm{~nm}$ in Fig. 3c, the emission wavelengths are relatively off resonance, compared with the metasurface with $D 212 \mathrm{~nm}$, and the optical signals are smaller than those from the metasurface with $D 212 \mathrm{~nm}$. It is thus most likely that the tuning at the emission wavelengths is secondary important to obtain the enhancing signals. Furthermore, the excitation wavelength is located at longer wavelength side than the peak of PlasPh resonance. This excitation condition is studied later with examining numerical results on the resonant electric-field distributions.

2.1.3 The role of the SAM. Here we discuss the role of the SAM, based on the experimental results in Fig. 2 and 3. It was confirmed in the previous study ${ }^{22}$ that the carboxy-end SAM cover on the entire Au outmost surface from the X-ray photoemission spectroscopy (XPS) analysis. Accordingly, we assume that the SAM plays a role to isolate the IR26 molecules from the Au surface.

Another feature in Fig. 3c is that Raman signals are hardly observed. Previously, it was reported that, when the outmost Au surface is covered with the carboxy-end SAM with the thickness more than $0.6 \mathrm{~nm}$, the Raman signals of fluorescent molecules emitting around $800 \mathrm{~nm}$ are efficiently suppressed. ${ }^{23} \mathrm{~A}$ similar effect was observed for the IR26 molecules. Indeed, Fig. 2 shows the prominent Raman signals on the PlasPh metasurface without SAM whereas Fig. 3 shows fluorescence-dominant signals on the PlasPh metasurfaces with the SAM. In the latter, the intrinsic LUMO-HOMO transition, that is, the fluorescent transition is considered to be efficiently preserved.

In the configurations without SAM (Fig. 2), both fluorescence and Raman scattering were observed. The excitation-relaxation dynamics in molecules yielding the optical signals in the artificial configurations has not been clarified to date. One

Table 1 Comparison of the SC PlasPh metasurfaces using Au and Ag. $\mathrm{FL}$ denotes fluorescence and Raman does Raman scattering. The enhancement factors (EFs) are described in the text

\begin{tabular}{lllll}
\hline Data & Metal & SAM & Enhanced signals & EFs \\
\hline Fig. 2 & $\mathrm{Au}$ & No & FL and Raman & $\sim 140$ \\
Fig. 3 & $\mathrm{Au}$ & Yes & FL & $\sim 50$ \\
Fig. 4 & $\mathrm{Ag}$ & No & FL & $\sim 20$
\end{tabular}

possibility to induce modification of the molecular eigen states allowing efficient Raman processes was inferred in a similar artificial configuration. ${ }^{23}$ Importantly, we are able to experimentally select the enhanced optical signals using the PlasPh metasurfaces.

Let us mention again that the optical signals are enhanced even when the SAM is not prepared on the PlasPh metasurfaces. Such enhancing effects on the PlasPh metasurfaces were observed using other fluorescent molecules working at and around the visible range. ${ }^{21-23,28}$ The quantitative discussion is provided later (Table 1). Metal-induced fluorescence quenching, which was often reported in other plasmonic platforms including flat metal surfaces ${ }^{29-31}$ has not been observed on the SC PlasPh metasurfaces. This is one of the advantages in the present PlasPh metasurfaces.

2.1.4 Ag SC PlasPh metasurfaces without SAM. Fig. 4 displays the results for Ag SC PlasPh metasurfaces. Fig. 4a shows the top-view SEM image of the Ag metasurface with $D$ $210 \mathrm{~nm}$. Fig. 4b illustrates the experimental configuration of the IR26 molecules on the outmost Ag surface without covering with SAM.

Fig. 4c shows the measured optical signals from the IR26 molecules dispersed on the Ag SC PlasPh metasurfaces (solid (a) Ag SC PlasPh

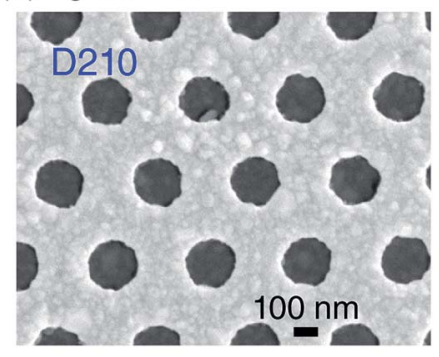

(b)

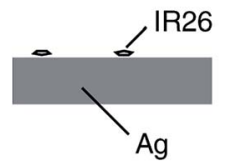

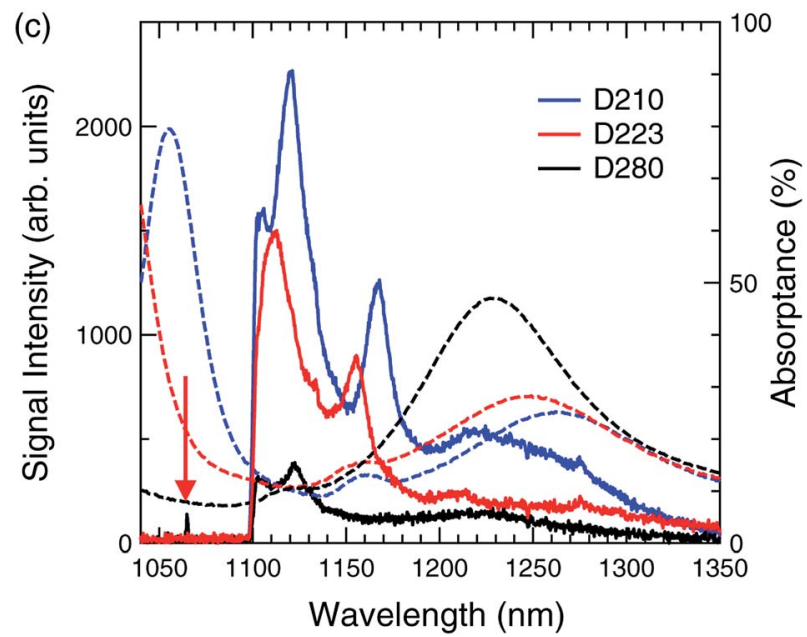

Fig. 4 (a) Top-view SEM image of the Ag PlasPh metasurface with airhole diameter $D 210 \mathrm{~nm}$. The scale bar indicates $100 \mathrm{~nm}$. (b) Schematic of the molecule configuration on the Ag PlasPh metasurface without SAM. (c) The measured optical signals (solid curves) and absorptance spectra (dashed curves). The blue, red, and black correspond to the Ag PlasPh metasurfaces with $D$ 210, 223, and $280 \mathrm{~nm}$, respectively. 
curves) and the absorptance spectra of the metasurfaces (dashed curves). Blue, red, and black curves denote the data on the $\mathrm{Ag}$ PlasPh metasurfaces with air-hole diameter $D$ 210, 223 and $280 \mathrm{~nm}$, respectively. The corresponding absorptance spectra were derived using eqn (1), based on the measured reflectance spectra in Fig. S4 (ESI $\dagger$ ). The red arrow indicates the excitation wavelength of $1064.1 \mathrm{~nm}$. For these Ag PlasPh metasurfaces, Raman signals were slightly observed (e.g., at 1275.8 $\mathrm{nm}$ ) whereas fluorescence was dominantly observed in an enhanced manner. Similarly to the Au SC PlasPh metasurfaces in Fig. 2 and 3, large absorptance at the excitation wavelength of $1064.1 \mathrm{~nm}$ is strongly correlated with the strong optical signals; moreover, the local maxima at the emission wavelengths contribute to the peaks of the observed signal peaks. Note that the observed peaks at 1167 and $1230 \mathrm{~nm}$ for the Ag PlasPh metasurface with $D 210 \mathrm{~nm}$ are associated with the local absorptance maxima at 1160 and $1260 \mathrm{~nm}$, respectively, and that the fluorescence spectrum of the IR26 molecules dispersed on a non-enhancing substrate decreases with increasing the wavelength (Fig. 1c). A similar tendency was observed for the Ag PlasPh metasurface with $D 223 \mathrm{~nm}$. Thus, the mechanism of enhancement is found qualitatively similar to the Au PlasPh metasurfaces.

Quantitatively, the optical signal intensities detected from the Ag PlasPh metasurfaces were smaller than those from the $\mathrm{Au}$ PlasPh metasurfaces; related to this result, we simply point out that the excitation wavelength was located at a longer wavelength than the absorptance peaks of the Ag PlasPh metasurfaces with $D 210$ and $223 \mathrm{~nm}$, and discuss this result later (Fig. 5 and 6) when examining the resonant electric fields.

In addition, although it is unclear why Raman-scattering components were slightly observed for the Ag PlasPh metasurfaces with any SAM, the quality of the deposited Ag may affect the observed signals; at least, the grain of Ag in Fig. 4a is larger than that of $\mathrm{Au}$ in Fig. 2b. Actually, we repeated the measurement by removing and depositing the Ag film; still, the results were almost the same. We consequently consider that, at least when using our preparation protocol and the deposition instrument, the results will remain unchanged. (a)

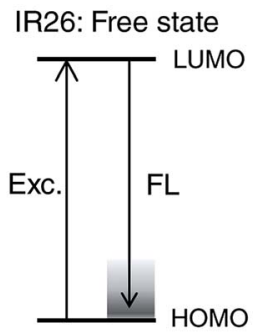

\section{(b) IR26 on SC metasurfaces}

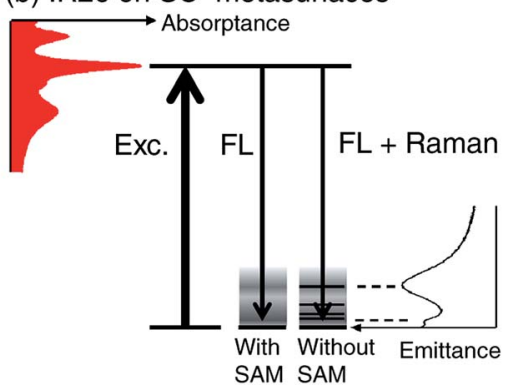

Fig. 5 Schematic energy diagrams of IR26 molecules. (a) Free state. FL denotes fluorescence. Excitation is abbreviated as Exc. (b) The energy diagrams of the IR26 molecules on the SC PlasPh metasurfaces, which is an artificial configuration for the dispersed molecules. Excitation is reinforced under the high absorptance. The optical signals are enhanced by the emittance.

\subsection{Mechanism of optical-signal enhancement}

Fig. 5 shows schematics of energy diagrams of IR26 molecules. Fig. 5a shows the energy diagram of free-state IR26 molecules; the diagram consists of HOMO-LUMO transition. The fluorescence (FL) has a band, shown with gray.

Fig. 5b depicts the enhanced transitions, based on the observed optical signals in the artificial configurations of the SC PlasPh metasurfaces. First, the excitation is reinforced (bold arrow) owing to the high absorptance of the SC metasurfaces. As seen in Fig. 2-4, when the excitation is tuned to the large absorptance peaks, the most prominent optical signals were observed; therefore, we consider that the resonance-tuning at the excitation wavelength is primarily important to obtain the large enhancement. The resonant electric fields are examined later (see Fig. 6 and 7). Second, the excited IR26 molecules have options in accordance with/without SAM; as shown in Fig. 3, the SC metasurfaces with SAM induce FL signals, whereas, as shown in Fig. 2, the SC metasurfaces without SAM dominantly induce FL and Raman scattering. Third, in both cases, the emitted optical signals were enhanced in accordance with the emittance of the SC metasurfaces (horizontal dashed lines in Fig. 5b); note that the emittance is equivalent to absorptance. ${ }^{26}$

\subsection{Comparison of metals}

In plasmonics, it is an open question to clarify the best metal for particular purposes. Here, from the viewpoint of the enhancing performance, we compare the noble metals used in the PlasPh metasurfaces.

Table 1 summarizes correlation of the metals and SAM, and the resultant enhanced effects. The thiolate SAM is suitable for growing on Au surface because of covalent bond $\mathrm{Au}-\mathrm{S}$; therefore, we used the SAM only for the Au PlasPh metasurfaces. The effect of the SAM was manifested in the appearance of Ramansignal components. The direct contact of the test molecules with the outmost Au surface enhances Raman signals as was observed in a previous study. ${ }^{23}$

As for the Ag PlasPh metasurfaces, the fluorescence was dominantly enhanced and the Raman-signal component was quite weak. Even when any SAM is not incorporated, the fluorescence is dominant on the $\mathrm{Ag}$ metasurfaces. This qualitative tendency is different from that in the $\mathrm{Au}$ metasurfaces.

Table 1 also lists enhancement factors (EFs), which were evaluated by taking the ratios of optical signal intensities measured for the SC PlasPh metasurfaces (Fig. 2d, 3c and 4c) to the optical signal intensity for the reference Si wafer in Fig. 1c. Other candidates, such as $\mathrm{SiO}_{2}$ substrate and flat $\mathrm{Au}$ film, for reference were also tested; however, they exhibited almost zero signal and turned out to be unsuitable for the reference; the measured data using a $\mathrm{SiO}_{2}$ substrate are shown in Fig. S5 $\left(\mathrm{ESI}^{\dagger}\right)$. The EFs are expressed ${ }^{22}$ such as

$$
\mathrm{EF}=\frac{N_{\mathrm{enh}}}{N_{0}} \frac{\eta_{\mathrm{enh}}}{\eta_{0}} \frac{\gamma_{\mathrm{enh}}(\mathbf{k})}{\gamma_{0}(\mathbf{k})}
$$

where $N$ denotes number of excited populations per unit time, $\eta$ does inner quantum efficiency, the ratio of $\gamma$ does purcell factor 
dependent on outgoing wavevector $\mathbf{k}$, and the subscripts enh and 0 stand for enhanced and reference states, respectively. We note that the $N$ is proportional to the electric-field intensity at the excitation and the $\gamma$ is proportional to that at the emission. The contribution by the emittance is also included in the factor $\gamma$. The $\eta$ reflects excited-state dynamics in the molecules and is responsible for the radiative transitions.

We list the EFs in Table 1, based on the most prominent peaks in Fig. 2d, 3c and 4c. The enhanced optical signals detected from the SC PlasPh metasurfaces exhibited spatial fluctuations by approximately $30 \%$ of the averaged values, as noted in the Section 4.1; therefore, the enhancement factors have large standard deviations and should be understood to represent the typical responses. This fluctuating property of enhancement factor is quite different from the previous studies using fluorescent molecules around the visible ranges ${ }^{22,23}$ because the enhancement of optical signals in those previous studies was almost uniform. Still, the enhancement factors, though not exact, serve as quantitative indicators and enable us to understand the extent of the enhancement.

In the present experiment, the $\mathrm{Au} \mathrm{SC}$ PlasPh metasurface without covering with SAM showed the best performance; for this metasurface, the enhancement was observed for both fluorescence and Raman signals. On the other hand, Au PlasPh metasurfaces with SAM and Ag PlasPh metasurfaces without SAM exhibit relatively smaller enhancement factors, as summarized in Table 1. This different enhancement effects cannot be understood only from the macroscopic optical properties of the $\mathrm{Au}$ and $\mathrm{Ag}$ PlasPh metasurfaces because the measured reflectance spectra are quite similar to each other, as shown in Fig. S2 and S4 (ESI $\dagger$ ). In the present sample preparation noted in Section 4.1, we can assume that the dispersed molecular density is almost equivalent in the three cases. In addition, the SAM layer is quite thin, being estimated to be less than $1 \mathrm{~nm} ;^{22}$ the electric-field enhancement is nearly independent of the SAM, as discussed later (Section 2.4). Taking account of these points, we suggest the following possibilities for the origin of the difference: (i) difference in contact (or absorption) state of the IR26 molecules on the metal atoms and the SAM, and (ii) difference of resonant electric fields according to the metal species. As for (ii), let us examine the resonant electric fields in the next subsection; see the discussion at the end of Section 2.4. As for (i), although we do not have experimental data nor the first-principle calculation data for analyzing a system of $10^{8}$ metal atoms and an IR 26 molecule, the possibility (i) is not excluded as one of the origins. Related to (i), we mention that the quality of the $\mathrm{Ag}$ film does not seem good enough (Fig. 4a) because the Ag grains are rather large, roughly $50 \mathrm{~nm}$ in diameter. Such Ag grains tend to have larger optical loss than crystalline $\mathrm{Ag}$ surfaces by molecular-beam-epitaxy growth..$^{32-34}$ Also, oxidation of the outmost Ag surface possibly affect the enhancement effect, in comparison with the outmost Au surface that is hardly oxidized.

The best performance of the Au PlasPh metasurface without SAM was realized at the condition in which the excitation wavelength was fairly well tuned to a high absorptance peak and the emission wavelengths were resonant with the local absorptance (i.e., emittance) maxima. Comparing the data of $D$ $212 \mathrm{~nm}$ (or $D 210 \mathrm{~nm}$ ) and $D 280 \mathrm{~nm}$ in Fig. 3 (or Fig. 4), it is most likely that the PlasPh resonance at the excitation wavelength primarily contributes to the enhancement of optical signals. As discussed on Fig. 3c above, the degree of resonance matching at the emission wavelengths is secondary important to enhance the optical signals. In the next subsection, we examine resonant electromagnetic (EM) fields, related to the optical-signal enhancement.

\subsection{Resonant EM fields on the SC PlasPh metasurfaces}

Fig. 6 compiles the numerical results for resonant EM fields in the excitation condition of IR26 molecules. Fig. 6a shows a computed absorptance spectrum; the air-hole diameter was assumed to be a typical value of $224 \mathrm{~nm}$ and the metal was set to be Au. The numbers 1 and 2 denote the first and second resonances at the normal incidence in the photon-energy order; note that the horizontal axis is represented with wavelength in accordance with the experimental results.

Fig. 6b presents the resonant $|\mathbf{E}|$ distribution (E: electricfield vector) at the peak wavelength of the second resonance, indicated by an arrow with $R$ in Fig. 6a; the capital letters $R, S$, and $L$ denote the resonant wavelength, shorter wavelength than the resonance, and longer wavelength, respectively. Representative $x y$ and $x z$ sections are shown in Fig. $6 \mathrm{~b}$ to visualize the absolute values of resonant electric field that contributes to exciting the IR26 molecules. The two $x y$ sections are top and bottom surfaces of the SC PlasPh metasurfaces; the $z$ positions are indicated in the $x z$ plane with dashed lines. The $x z$ section was taken through the centers of air holes. The incidence was
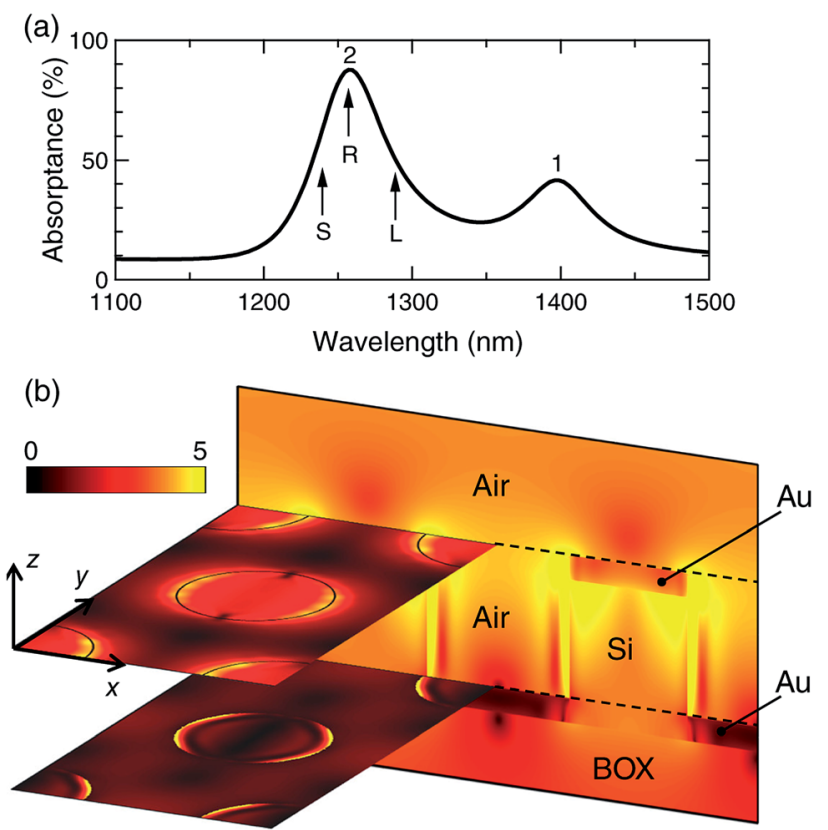

Fig. 6 Resonant EM fields. (a) Numerically calculated absorptance spectrum at the normal incidence. (b) The $|\mathrm{E}|$ distribution at a resonant wavelength $R$ in (a). Representative $x y$ and $x z$ planes are shown, on which the IR26 molecules are mainly dispersed. 
set to $|\mathbf{E}|=1$. Although the maximal $|\mathbf{E}|$ value of the resonant electric field appears on the $x z$ plane and takes 38.7, we display the $|\mathbf{E}|$ distribution in the $(0,5)$ range with the linear scale, since almost all the locations except for the hot (or the $|\mathbf{E}|$-largest) spots become dark and cannot be seen. The hot spots appear at the interface between the top $\mathrm{Au}$ and Si. Fig. $6 \mathrm{~b}$ shows that afew-fold enhanced electric fields locate on the top $\mathrm{Au}$, near the air holes, and inside the air holes, implying that electricfield enhancement is not so large. Thus, Fig. 6b does not support that the prominently enhanced signals summarized in Table 1 are attributable only to the electric-field enhancement. This numerical insight is consistent with the previously reported results employing the Au SC PlasPh metasurfaces. ${ }^{21-23}$

Let us further examine the enhanced optical signals and resonant electric fields. Fig. 3c shows a definite difference under the excitation at the second resonance; the two cases $(D 212 \mathrm{~nm}$, blue; $D 220 \mathrm{~nm}$, red) yield strong and weak optical signals, respectively. As far as we assume the experimental conditions are equivalent, the difference is attributable to relative excitation-wavelength positions for the second PlasPh resonance peak around $1060 \mathrm{~nm}$. The SC PlasPh metasurface with $D$ $212 \mathrm{~nm}$ was excited at a shorter wavelength than the second resonance whereas the metasurface with $D 220 \mathrm{~nm}$ was excited at a longer wavelength. To clarify the difference, we compared electric-field intensity $|\mathbf{E}|^{2}$ distributions at the wavelengths $S, R$, and $L$ around the second resonance in Fig. 5a; the $|\mathbf{E}|^{2}$ distributions are shown in the next.

Fig. 7 shows resonant electric-field distributions in the $x z$ sections. The intensity $|\mathbf{E}|^{2}$ is plotted in color on the $x z$ section through the centers of the air holes. The section in Fig. $7 \mathrm{~b}$ is identical to the $x z$ section in Fig. 6b. Note that, in Fig. 6b, absolute value of electric field $|\mathbf{E}|$ is plotted in the linear scale whereas the intensity distributions in Fig. 7 are shown with the logarithmic scale for $(0.1,400)$ range in saturated manners for better visibility. The intensities exceeding 400 in Fig. 7 are represented with yellow. The maxima intensities are 741, 1498 and 1125 in Fig. 7a-c, respectively.

The resonant wavelengths of Fig. 7a-c are indicated by the arrows $L, R$ and $S$ in Fig. 5a, respectively. When comparing the electric-field intensities at the interface of air/Au on the top layer, it turns out that the PlasPh resonance has relatively strong electric field at the resonant and shorter wavelengths; at the longer wavelengths, the electric-field intensity takes relatively small values. This qualitative tendency agrees with the enhancement factors in Table 1.

In Fig. 7, it is also verified that the hot spots corresponding to large intensity exceeding 100 are localized at the side of air holes and that dark spots, which look black, appear on the top Au surface in Fig. 7a and on the bottom Au surfaces in Fig. 7a-c. The dispersed IR26 molecules are considered to be placed mainly on the Au surfaces and possibly on the Si sidewall. Nevertheless, the contribution from the Si sidewall is most likely to be small because, if it should be dominant, the observed optical-signal intensities would show similar amounts at the three conditions of $L, R$ and $S$ in Fig. 5a; however, the differences in the maximum electric-field intensity are about within a factor of 2 , as mentioned above, whereas the difference

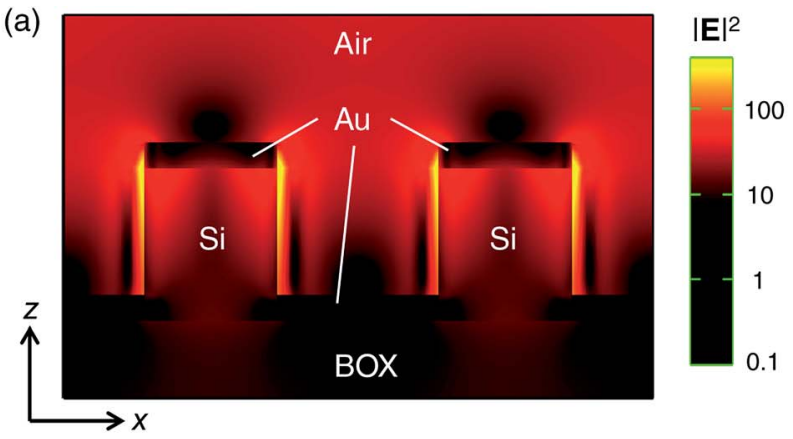

(b)

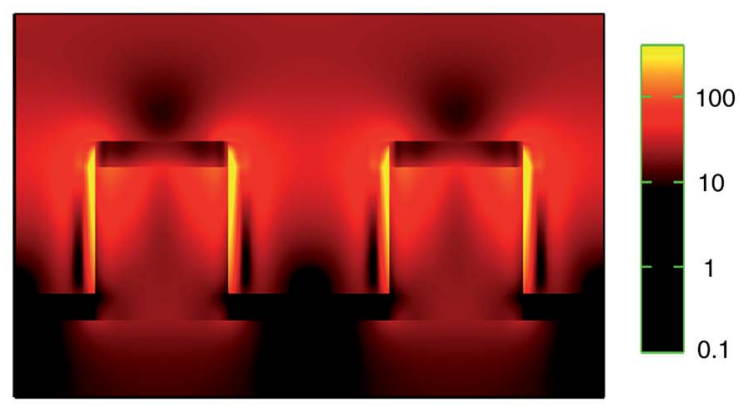

(c)

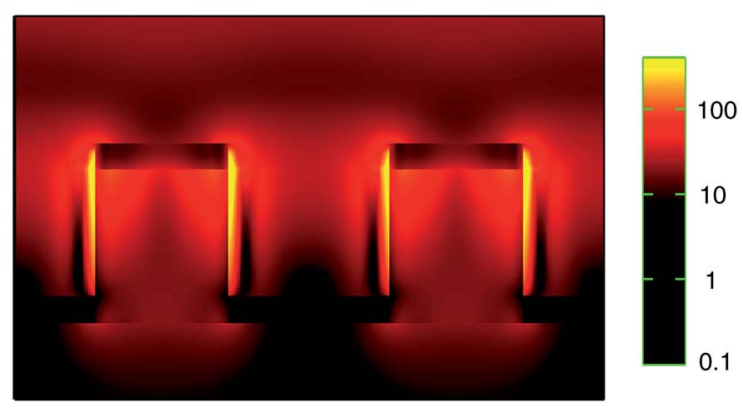

Fig. 7 Resonant electric-field distributions. The intensity $|\mathrm{E}|^{2}$ is shown in common with the logarithmic scale for $(0.1,400)$ range. (a)-(c) correspond to the resonant wavelengths indicated in Fig. 5a by arrows $L, R$, and $S$, respectively. These panels are presented in saturated manners; see the text for more details.

in the enhanced optical-signal intensities is more than 10 fold (Fig. 3c). Thus, we can conclude that the molecules dispersed on the top layer mostly contribute to the enhanced optical signals. We also found that the excitation efficiency of the IR26 molecules is high at the $R$ and $S$ conditions (i.e., Fig. 7b and c) and becomes smaller at the $L$ condition (i.e., Fig. 7a).

The intensity distributions indicate that the excitation at the longer wavelength, corresponding to $L$, is associated with dark spots on the top and bottom of the Au surface, meaning that the excitation efficiency of the IR26 molecules is relatively lower than the excitation at the $S$ position. Indeed, the excitation at the $L$ position results in smaller optical signals than the excitation at the $S$ position (Fig. 3). ${ }^{35}$ As for the Ag cases in Fig. 4, both Ag SC PlasPh metasurfaces of $D 210$ and $D 223 \mathrm{~nm}$ were excited at the $L$ positions; consequently, they exhibited quantitatively similar optical signals to each other. Thus, these numerical results in Fig. 7 consistently describe all the observed optical-signal intensity ratios in this paper, strongly suggesting that it is important to tune the PlasPh resonance to the 
excitation wavelength. The SC PlasPh metasurfaces are tunable at the resonant wavelengths by varying the air-hole diameters and are advantageous because the SC metasurfaces are applicable for a variety of molecules that have different HOMOLUMO transition wavelengths.

At the end of discussion, we address the origin of the different enhancement factors noted in Section 2.3. The possibility (ii) is attributed to the different resonant effects owing to the metal species. In this subsection, we have seen the resonant electric fields in the Au PlasPh metasurface. Resonant electric fields in a structurally equivalent $\mathrm{Ag}$ PlasPh metasurface are shown in Fig. S6 (ESI†); as a result, we confirm that the resonant electric fields in the $\mathrm{Ag}$ metasurface are quite similar to those in the Au metasurface (Fig. 7). Therefore, we can exclude the possibility (ii) as origin of the different enhancement factors in the $\mathrm{Au}$ and $\mathrm{Ag}$ cases.

\section{Conclusions}

We have shown artificially enhanced optical signals emitted from the IR26 molecules dispersed on the SC PlasPh metasurfaces with high-emittance resonances. The enhancement was prominent and clearly observed in comparison with the reference signal detected on a non-enhancing Si substrate. We moreover showed that the enhanced optical signals are selected by choosing the outmost surface with/without SAM. This strategy was first demonstrated for fluorescent molecules working around the visible range $\mathrm{e}^{23}$ and has been validated for the fluorescent molecules in the living-body window. Thus, the SC PlasPh metasurfaces are substantiated to serve as quite versatile sensors for optical detections of fluorescent molecules from the visible to the near-infrared $1.2 \mu \mathrm{m}$ ranges. It was also clarified, by numerically examining the resonant electric-field distributions, that it is important to tune the excitation wavelength to the resonance wavelengths of the SC PlasPh metasurfaces for significant enhancement of the optical signals.

\section{Methods}

\subsection{Preparation of metasurfaces}

Nanopatterns for the metasurfaces were produced by ultraviolet (UV) NIL in a high-throughput manner. Nanohole arrays of $1 \times$ $1 \mathrm{~cm}^{2}$ dimensions were introduced on SOI substrates (SOI 200 $\mathrm{nm} / \mathrm{BOX} 375 \mathrm{~nm} / \mathrm{Si}$ wafer $625 \mu \mathrm{m}$ ). The array periodicity was $410.5 \mathrm{~nm}$ and the nanoholes were arrayed in a hexagonal manner. The detailed nanofabrication procedure was reported previously. ${ }^{28}$ We deposited Au or Ag on the nanopatterned SOI substrates as normal as possible to the substrates, and obtained the SC metasurfaces in accordance with the design (Fig. 2a). The thickness of $\mathrm{Au}$ and $\mathrm{Ag}$ was set to $35 \mathrm{~nm}$, which is thin enough to separate the top and bottom layers, and is thick enough to induce plasmonic resonances. The fabricated structures were confirmed to take section-view SEM images; a typical image ${ }^{28}$ is cited in Fig. S1 (ESI†). The SC metasurfaces exhibit a series of PlasPh resonances that have been clarified recently. ${ }^{19}$ In the present experiment, the second resonance at the normal incidence mainly contributed to enhancing the optical signals.
One advantage of the nanoimprinted SOI substrates is their reusability. We used SC PlasPh metasurfaces in the fluorescentmolecule experiment, removed the noble metals by wet etching, and recovered them as SC PlasPh metasurfaces by performing the metal deposition. This reusability substantially expedited the optical experiment.

The IR26 methanol solution was $50 \mu \mathrm{M}$, which was prepared by adjusting the density using a more dense solution in Fig. 1 a. We dispersed a $2 \mu \mathrm{L}$ drop of the solution on each SC PlasPh metasurface. The drop promptly spread to a large area (i.e., approximately $2 \mathrm{~cm}$ diameter) and became dry in air within a few seconds. When we dispersed the molecules, we did not observe any explicit difference with/without the SAM; therefore, we consider that the molecules were dispersed similarly to each other.

If we assume that the molecules were dispersed in an ideally uniform way, we can estimate the molecule density to be 1 molecule $/(2.6 \times 2.6) \mathrm{nm}^{2}$ on the surface of SC PlasPh metasurface with $D 212 \mathrm{~nm}$, where the air-hole wall is included. Thus, the idealized density turns out to be sparse enough to isolate each molecule.

As noted in the Section 2, it is difficult to completely dissolve IR26 molecules in organic solvents. Therefore, we can easily infer that the dispersed molecules do not obey the ideally spatial distribution assumed in the preceding paragraph. Indeed, we observed inhomogeneous responses of the opticalsignal intensity for the SC PlasPh metasurfaces though the spectral shapes were virtually the same. In our data, the fluctuations were approximately $30 \%$ of average values. This point was referred to in estimating the EFs in Table 1. In Fig. 2-4, we show measured typical optical signals, except for the largest and smallest data; note that the averaged signals are no longer measured data because they underwent the calculating procedure.

\subsection{Optical measurement}

Reflectance spectra were measured using a spectrometer that enables to conduct incidence-angle resolved measurements. Reflectance was measured at the incidence angle of $5^{\circ}$, as a substitute for the normal incidence. The incident polarization was $p$ polarization, for which the incident electric-field vector is kept in the $x z$ plane of Fig. 2a when the plane of incidence is the $x y$ plane. At the nearly normal incidence, the measurement under $s$ polarization, for which the incident electric-field vector is parallel to the $y$ axis in Fig. 2a when the plane of incidence is the $x y$ plane, resulted in almost identical reflectance spectra. The light absorptance spectra in Fig. 2-4 were evaluated using eqn (1) and the measured reflectance spectra.

We mention that all the measured reflectance spectra shown in this paper were measured before dispersing the IR26 molecules. The dispersed density was rather small; consequently, we did not observe any detectable change in the reflectance spectra due to the molecule dispersion.

The enhanced optical signals from the IR26 molecules dispersed on the SC PlasPh metasurfaces were measured in an illumination-and-collection setup. An objective lens $(\times 10$, 
numerical aperture 0.26) focused the excitation laser light on the SC PlasPh metasurfaces and collected the optical signals emitted by the IR26 molecules. The incidence and collection were carried out for the nearly normal incidence in accordance with the numerical aperture. The excitation wavelength was $1064.1 \mathrm{~nm}$ and the light was emitted by a single-mode continuous-wave laser. The excitation power was $12 \mathrm{~mW}$ on the sample surfaces and the data acquisition time was $10 \mathrm{~s}$. A setup similar to the one in this study was previously reported with the configuration diagram. ${ }^{23}$

\subsection{Numerical implementation}

Numerical calculations for optical spectra and EM fields were conducted using rigorous coupled-wave analysis (RCWA), ${ }^{36}$ which enables to numerically solve Fourier-transformed Maxwell equations and is one of the first-principle techniques in electromagnetism. To evaluate stacked structures with avoiding numerical divergence, scattering-matrix algorithm ${ }^{37}$ has to be incorporated. Numerical code based on the RCWA and scattering-matrix algorithm was prepared and executed on supercomputers in a multi-parallel implementation manner.

In the RCWA-based computations, we used measured complex relative permittivities of $\mathrm{Au}$ and $\mathrm{Ag}$ as the material parameters. The measured data are shown in Fig. S6 (ESI + ). The reflectance spectra that were numerically calculated using measured permittivities show better correspondence with the measured optical spectra than the numerical results based on the literature data. ${ }^{38}$ Therefore, in this study, we adopted measured permittivities. In a recent study on hightransmittance metasurfaces, ${ }^{39}$ we also found that the measured permittivities result in better reproduction of experimental data.

As for other materials involved in the SC PlasPh metasurfaces, we used material parameters in literature and typical values. Complex refractive index of $\mathrm{Si}$ was taken from literature. ${ }^{40}$ The refractive index of the BOX layer was set to 1.46 since the material is $\mathrm{SiO}_{2}$. The refractive index of air was set to 1.00027 .

\section{Conflict of interest}

There are no conflicts of interest to declare.

\section{Acknowledgements}

We thank Bongseok Choi for producing nanoimprinted substrates based on SOI substrates. M. I. thanks KAKENHI Grant (Number 26706020) from the Japan Society for Promotion of Science and HPCI System Research Project (IDs: hp160035, hp170134) from Research Organization for Information Science and Technology, Japan; the numerical implementations were conducted at Cyberscience Center, Tohoku University. This study was partially supported by the 4 th mid-term project in NIMS. Nanofabrication was conducted at NanoIntegration Platform in NIMS.

\section{Notes and references}

1 J. R. Lakowicz, K. Ray, M. Chowdhury, H. Szmacinski, Y. Fu, J. Zhang and K. Nowaczyk, Analyst, 2008, 133, 1308-1348.

2 M. Bauch, K. Toma, M. Toma, Q. Zhang and J. Dostalek, Plasmonics, 2014, 9, 781-799.

3 D. L. Jeanmaire and R. P. van Duyne, J. Electroanal. Chem., 1977, 84, 1-20.

4 M. G. Albrecht and J. A. Creighton, J. Am. Chem. Soc., 1977, 99, 5215-5217.

5 A. M. Glass, P. F. Liao, J. G. Bergman and D. H. Olson, Opt. Lett., 1980, 5, 368-370.

6 J. F. Li, Y. F. Huang, Y. Ding, Z. L. Yang, S. B. Li, X. S. Zhou, F. R. Fan, W. Zhang, Z. Y. Zhou, D. Y. Wu, B. Ren, Z. L. Wang and Z. Q. Ti, Nature, 2010, 464, 392-395.

7 Y. Li, Q. Shi, P. Zhang, Y. Xiahou, S. Li, D. Wang and H. Xia, J. Mater. Chem. C, 2016, 4, 6649-6656.

8 D. R. Nayak, N. Bhat, M. Venkatapathi and S. Umapathy, J. Mater. Chem. C, 2017, 5, 2123-2129.

9 A. Kinkhabwala, Z. Yu, S. Fan, Y. Avlasevich, K. Müllen and W. E. Moerner, Nat. Photonics, 2009, 3, 654-657.

10 M. Schmelzeisen, Y. Zhao, M. Klapper, K. Müllen and M. Kreiter, ACS Nano, 2010, 4, 3309-3317.

11 C.-C. Fu, G. Ossato, M. Long, M. A. Digman, A. Gopinathan, L. P. Lee, E. Gratton and M. Khine, Appl. Phys. Lett., 2010, 97, 203101.

12 W. Zhang, F. Ding, W.-D. Li, Y. Wang, J. Hu and S. Y. Chou, Nanotechnology, 2012, 23, 225301.

13 L. Zhou, F. Ding, H. Chen, W. Ding, W. Zhang and S. Y. Chou, Anal. Chem., 2012, 84, 4489-4495.

14 S. Dinda, V. Suresh, P. Thoniyot, A. Balčytis, S. Juodkazis and S. Krishnamoorthy, ACS Appl. Mater. Interfaces, 2015, 7, 27661-27666.

15 J. Pang, I. G. Theodorou, A. Centeno, P. K. Petrov, N. M. Alford, M. P. Ryan and F. Xie, J. Mater. Chem. C, 2017, 5, 917-925.

16 Y. Nishijima, Y. Hashimoto, L. Rosa, J. B. Khurgin and S. Juodkazis, Appl. Phys. A, 2014, 117, 647-650.

17 I. G. Theodorou, Z. A. R. Jawad, H. Qin, E. O. Aboagye, A. E. Porter, M. P. Ryan and F. Xie, Nanoscale, 2016, 8, 12869-12873.

18 G. Hong, S. M. Tabakman, K. Welsher, H. Wang, X. Wang and H. Dai, J. Am. Chem. Soc., 2010, 132, 15920-15923.

19 M. Iwanaga and B. Choi, Nano Lett., 2015, 15, 1904-1910.

20 M. Iwanaga, Plasmonic Resonators: Fundamentals, Advances, and Applications, Pan Stanford Publishing, Singapore, 2016.

21 M. Iwanaga, B. Choi, H. T. Miyazaki, Y. Sugimoto and K. Sakoda, J. Nanomater., 2015, 2015, 507656.

22 B. Choi, M. Iwanaga, H. T. Miyazaki, Y. Sugimoto, A. Ohtake and K. Sakoda, Chem. Commun., 2015, 51, 11470-11473.

23 M. Iwanaga, B. Choi, H. T. Miyazaki and Y. Sugimoto, Nanoscale, 2016, 8, 11099-11107.

24 B. Choi, M. Iwanaga, T. Ochiai, H. T. Miyazaki, Y. Sugimoto and K. Sakoda, Appl. Phys. Lett., 2014, 105, 201106.

25 A. Seilmeier, B. Sens, K. H. Drexhage and W. Kaiser, Opt. Lett., 1983, 8, 205-207. 
26 J.-J. Greffet and M. Nieto-Vesperinas, J. Opt. Soc. Am. A, 1998, 15, 2735-2744.

27 J. C. Love, L. A. Estroff, J. K. Kriebel, R. G. Nuzzo and G. M. Whitesides, Chem. Rev., 2005, 105, 1103-1170.

28 B. Choi, M. Iwanaga, H. T. Miyazaki, K. Sakoda and Y. Sugimoto, J. Micro/Nanolithogr., MEMS, MOEMS, 2014, 13, 023007.

29 R. R. Chance, A. H. Miller, A. Prock and R. Silbey, J. Chem. Phys., 1975, 63, 1589-1595.

30 E. Dulkeith, A. C. Morteani, T. Niedereichholz, T. A. Klar, J. Feldmann, S. A. Levi, F. C. J. M. van Veggel, D. N. Reinhoudt, M. Möller and D. I. Gittins, Phys. Rev. Lett., 2002, 89, 203002.

31 P. Anger, P. Bharadwaj and L. Novotny, Phys. Rev. Lett., 2006, 96, 113002.

32 J. H. Park, P. Ambwani, M. Manno, N. C. Lindquist, P. Nagpal, S.-H. Oh, C. Leighton and D. J. Norris, $A d v$. Mater., 2012, 24, 3988-3992.
33 Y.-J. Lu, J. Kim, H.-Y. Chen, C. Wu, N. Dabidian, C. E. Sanders, C.-Y. Wang, M.-Y. Lu, B.-H. Li, X. Qiu, W.-H. Chang, L.-J. Chen, G. Shvets, C.-K. Shih and S. Gwo, Science, 2012, 337, 450-453.

34 Y. Wu, C. Zhang, N. M. Estakhri, Y. Zhao, J. Kim, M. Zhang, X.-X. Liu, G. K. Pribil, A. Alù, C.-K. Shih and X. Li, Adv. Mater., 2014, 26, 6106-6110.

35 Similar tendency was observed for the Au SC PlasPh metasurfaces without SAM though the data are not shown.

36 L. Li, J. Opt. Soc. Am. A, 1997, 14, 2758-2767.

37 L. Li, J. Opt. Soc. Am. A, 1996, 13, 1024-1035.

38 A. D. Rakić, A. B. Djurušić, J. M. Elazar and M. L. Majewski, Appl. Opt., 1998, 37, 5271-5283.

39 H. Kurosawa, B. Choi, Y. Sugimoto and M. Iwanaga, Opt. Express, 2017, 25, 4446-4455.

40 E. D. Palik, Handbook of Optical Constants of Solids II, Academic, San Diego, USA, 1991. 